\title{
Improved calibration of vertical scanning optical profilometers for spherical profiles measurements
}

Jeremy Béguelin, Toralf Scharf, Wilfried Noell, Reinhard Voelkel

Jeremy Béguelin, Toralf Scharf, Wilfried Noell, Reinhard Voelkel, "Improved calibration of vertical scanning optical profilometers for spherical profiles measurements," Proc. SPIE 10692, Optical Fabrication, Testing, and Metrology VI, 1069207 (15 June 2018); doi: 10.1117/12.2306797 


\title{
Improved calibration of vertical scanning optical profilometers for spherical profiles measurements
}

\author{
Jeremy Béguelin ${ }^{1}$, Toralf Scharf ${ }^{2}$, Wilfried Noell ${ }^{1}$, and Reinhard Voelkel ${ }^{1}$ \\ ${ }^{1}$ Süss MicroOptics SA, 2068 Hauterive, Switzerland \\ ${ }^{2}$ Nanophotonics and Metrology Laboratory, École Polytechnique Fédérale de Lausanne, \\ 1015 Lausanne, Switzerland
}

\begin{abstract}
A new method for calibrating optical scanning profilometers is presented. Especially adapted to spherical and aspherical profile measurements, it shows an increase of accuracy bigger than one order of magnitude for radius of curvature measurements. Calibration of vertical scaling is obtained with a reduction of its uncertainty by a factor larger than 2, which also demonstrates the advantage of this method for any surface measurements. Using commercially available reference balls, this method is easily implementable.
\end{abstract}

Keywords: Profilometer calibration, Microball, Microlens, Micro-optics metrology, Confocal microscopy, Coherence scanning interferometry

\section{INTRODUCTION}

Spherical and aspherical surfaces are of great importance in optics. Fabricating high quality surfaces is challenging, especially at the microscale level. Fabrication process optimization requires continuous feedback and the manufactured surfaces therefore need to be measured. In particular, the deviation from the target surface has to be quantified in order to minimize it. In micro-optics, this characterization is usually done with optical scanning profilometers such as confocal microscopes and coherence scanning interferometers (CSIs). For spherical surfaces, the radius of curvature (ROC) defines the surface whereas aspherical surfaces additionally require the conic constant $\kappa$. Measuring these parameters with high level of confidence is crucial for process optimization. The level of accuracy which can be obtained is directly linked with the profilometer calibration. Normally, this calibration is achieved using step standards and periodic structure standards to calibrate the vertical motion and the magnification, respectively [1]. For state of the art micro-optical applications, the uncertainties of the standards can be as large as the manufacturing tolerance. To solve this issue, a new calibration method is proposed. It consists in using a reference ball, whose global ROC is known with very high accuracy. Measuring the surface of this ball with the optical profilometer at random locations, similarly to what is done in wavefront calibration for interferometers [2], provides a distribution of local ROCs whose average coincides with high confidence to the certified ROC of the ball when the profilometer is calibrated. Thereby, the ratio between uncalibrated local ROC distribution and the certified ROC gives important calibration information. With this approach, the uncertainty of ROC and conic constant measurements can be reduced to up to one order of magnitude.

\section{METHODS}

Optical scanning profilometers such as confocal microscopes or CSI record multiple 2D images while scanning along the vertical direction. This allows the reconstruction of a surface on a certain area defined by the field of view (FOV) of the microscope objective (MO). The magnification of the objective provides the lateral $(x, y)$ scaling whereas the scanning actuator - generally a piezo - gives the vertical one. In order to correctly measure the surface, the calibration of the magnification and of the vertical motion is of central importance. Usually, the determination of the vertical calibration factor is done by measuring a reference step whereas the measurement of periodic structures gives the lateral calibration factor. By definition, the vertical calibration factor $\alpha$ is given by $z^{\prime}=\alpha z$, where ' denotes the uncalibrated $\mathrm{z}$ coordinate. Respectively, the lateral calibration factor $\beta$ is defined by $x^{\prime}=\beta x$ and $y^{\prime}=\beta y$.

Further author information: E-mail: jeremy.beguelin@suss.com

Optical Fabrication, Testing, and Metrology VI, edited by Sven Schröder, Roland Geyl, Proc. of SPIE

Vol. 10692, $1069207 \cdot$ @ 2018 SPIE · CCC code: 0277-786X/18/\$18 · doi: 10.1117/12.2306797 
Any aspherical surface can be expressed in cartesian coordinates as [3]

$$
z(r)=\frac{r^{2}}{R\left(1+\sqrt{1-(1+\kappa) \frac{r^{2}}{R^{2}}}\right)},
$$

where $R$ is the asphere ROC and $r=\sqrt{x^{2}+y^{2}}$ the radial coordinate. For an uncalibrated system, the equation becomes

$$
z^{\prime}\left(r^{\prime}\right)=\frac{\alpha}{\beta^{2}} \frac{r^{\prime 2}}{R\left(1+\sqrt{1-(1+\kappa) \frac{1}{\beta^{2}} \frac{r^{\prime 2}}{R^{2}}}\right)}=\frac{r^{\prime 2}}{R^{\prime}\left(1+\sqrt{1-\left(1+\kappa^{\prime}\right) \frac{r^{\prime 2}}{R^{\prime 2}}}\right)} .
$$

An asphere is thus transformed into another asphere. By identification, the uncalibrated ROC is given by

$$
R^{\prime}=\frac{\beta^{2}}{\alpha} R \stackrel{\text { def }}{=} \gamma R
$$

Likewise, the uncalibrated conic constant $\kappa^{\prime}$ is given by

$$
\kappa^{\prime}=\frac{\beta^{2}}{\alpha^{2}}(\kappa+1)-1
$$

The measurement of the uncalibrated ROC provides the ratio between $\beta^{2}$ and $\alpha$, what we call $\gamma$. It is the only calibration factor when measuring the ROC of aspherical surfaces. For a calibrated system, the uncertainty in the ROC measurement is given by the uncertainty of the calibration factors, which is at least the uncertainty of the certified standards. The values of $u(\alpha)$ and $u(\beta)$ are taken from traceable standards provided by national institutes of metrology such as NIST or METAS. They correspond to the standard uncertainty [4]. This leads to

$$
\frac{u(R)}{R}=\sqrt{\left(\frac{u(\alpha)}{\alpha}\right)^{2}+2\left(\frac{u(\beta)}{\beta}\right)^{2}}=0.27 \% .
$$

In the same way, the uncertainty of the conic constant is

$$
u(\kappa)=2(1+\kappa) \sqrt{u(\alpha)^{2}+u(\beta)^{2}},
$$

which is a function of the conic constant itself. For the spherical case, $u(\kappa)=0.0052$.

If only spherical surfaces are considered, the conic constant is set to 0 and is no longer a parameter. The ROC of the best sphere fit is thus different from the ROC of the best asphere fit, as well as their uncertainties. In a certain way, the uncertainty of the conic constant is added to the best sphere fit ROC uncertainty. In this case, it depends on the ROC and on the FOV. Figure 1 shows the ROC uncertainty which has to be added when going from aspherical to spherical surfaces. Combinations of ROC and FOV are taken to be physically measurable in reflection by the profilometer. This uncertainty becomes larger when ROC $\sim$ FOV. However, spherical microlenses fulfilling this condition have a large amount of spherical aberration and should not be designed without a conic constant. The maximal uncertainty of the ROC becomes $0.28 \%$ in the worse case.

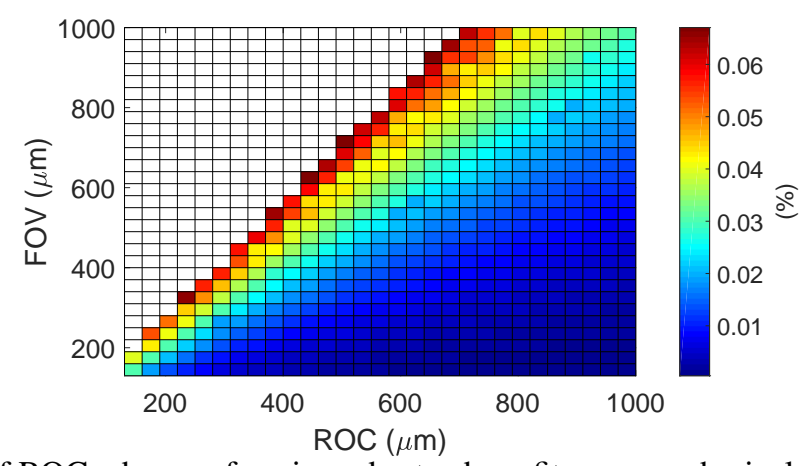

Figure 1: Uncertainty of ROC when performing a best sphere fit on an aspherical surface, $u(\kappa)=0.0052$. 
Usually, allowed tolerances on microlenses ROC can reach $\pm 1.5 \%$, which is about 6 times the derived standard uncertainty. This is problematic since a large part of the tolerance is taken away by the characterization and little tolerance remains for the fabrication process. This invariably decreases the production yield. To overcome this limitation, the use of a reference ball to obtain the calibration factor $\gamma$ is proposed. The certified ROC uncertainty of such a ball is in the order of $0.007 \%$, which is 40 times less than the uncertainty derived for ROC measurements. However, this certified uncertainty cannot be directly applied to ROC measurements which are defined only on the FOV area of the MO. These local ROCs are indeed different from the global ROC since the ball is not perfect. However, simulations allow to link local ROCs measured with optical scanning profilometer to the global ROC to benefit from the small uncertainty of the global ROC of the ball.

\section{SIMULATIONS}

The idea is to simulate a non-ideal reference ball which corresponds to the real one and to simulate ROC measurements by optical profilometers at different locations on it. The comparison between the local ROC distribution and the global ROC allows an accurate determination of the calibration factor $\gamma$.

The real ball is represented by adding a sum of orthogonal polynomials to the perfect ball. In spherical coordinates, the surface of a real ball can be written [5] as

$$
\rho(\theta, \phi)=R_{0}+\sum_{l} \sum_{m} a_{l, m} Y_{l, m}(\theta, \phi)
$$

with $Y_{l, m}$ the spherical harmonics and $a_{l, m}$ their corresponding coefficients. The key step is to choose the unknown coefficients $a_{l, m}$ to represent the real ball accurately. The simulated ball must give similar values in comparison with the real ball for two quantities. The first one is the sphericity error which is defined as the peak-to-valley value of the deviation from the best fit sphere. The second one is the local ROC distribution which is obtained experimentally from the real ball. To fulfill these conditions, the function

$$
a_{l, m}=a_{0}(l, m) e^{-(l+|m|) / N}
$$

is used. The coefficients $a_{0}(l, m)$ and $N$ are free parameters.

Local ROC measurements are simulated to correspond to measurements performed with the profilometer. This means randomly selecting a reduced area of the size of the FOV, and fitting Eq. 1 to obtain the ROC at this location. Figure 2 shows the local ROC distribution for 1000 simulated surface measurements at different locations of a simulated ball. Global ROC $=700 \mu \mathrm{m}$, the amplitudes $a_{0}(l, m)$ are random numbers equally distributed between 0 and $0.025 \mu \mathrm{m}$ and $\mathrm{N}$ is equal to 22 . The sphericity error is $100 \mathrm{~nm}$. The average of this distribution is $700.002 \mu \mathrm{m}$ and the standard deviation is $0.25 \mu \mathrm{m}$. This shows that for almost perfect balls, the average of the local ROC distribution coincides with a high accuracy to the global ROC. This thus links local ROC measurements at random locations to the global certified ROC.

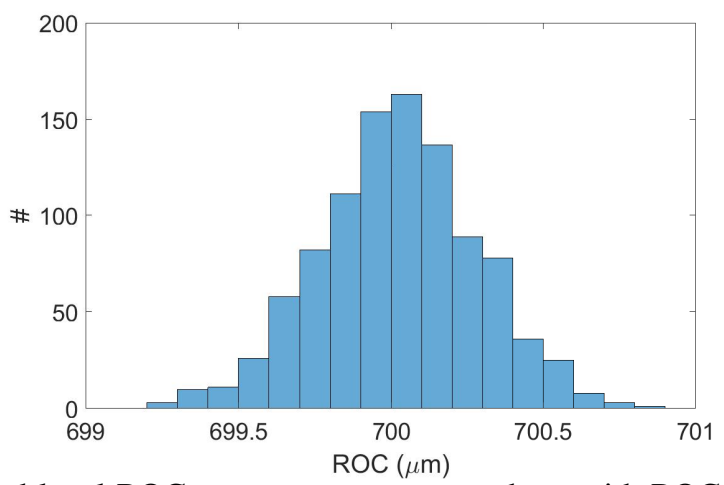

Figure 2: Distribution of simulated local ROC measurements on a sphere with $\mathrm{ROC}=700 \mu \mathrm{m}$ and a sphericity error of $100 \mathrm{~nm}$. 


\section{CALIBRATION EXAMPLE}

As an example, a ruby ball with a global ROC of $700 \mu \mathrm{m}$ is used to obtain the calibration factor $\gamma$ for the 50x MO of a CSI. So far, the repeatability of the profilometer has not been considered. Indeed, the repeatability of the tool makes one measurement of a local ROC a random variable. The measured local ROCs distribution is consequently the convolution of the repeatability distribution and the local ROCs distribution. In order to take into account this effect, the repeatability of the tool is characterized by measuring one local ROC 50 times in a row. Results are presented in Fig. 3.

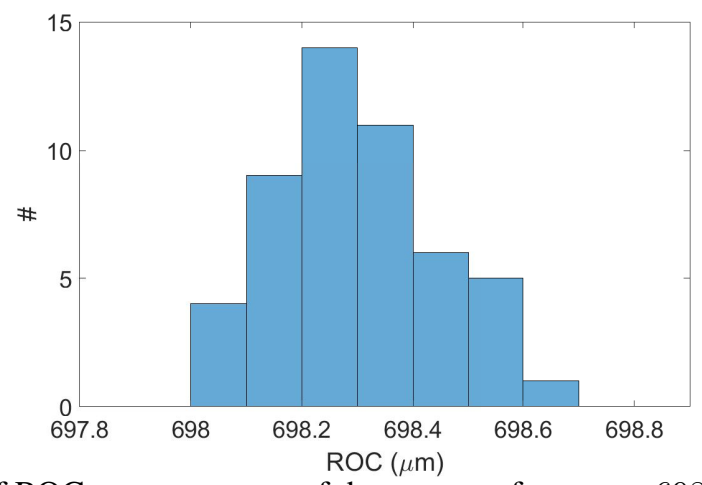

Figure 3: Distribution of ROC measurements of the same surface: $\mu=698.29 \mu \mathrm{m}$ and $\sigma=0.15 \mu \mathrm{m}$.

The distribution is centered at $698.29 \mu \mathrm{m}$ and has a standard deviation of $0.15 \mu \mathrm{m}$. By modeling this distribution with a Gaussian distribution, it is possible to perform the deconvolution in order to get the real local ROCs distribution.

The local ROCs distribution is then obtained by measuring the local ROC at 50 randomly chosen locations. The distribution of the local ROCs is presented in Fig. 4.

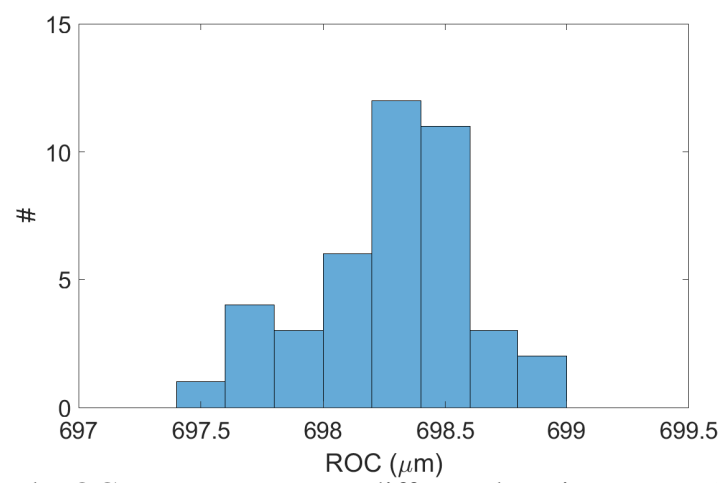

Figure 4: Distribution of local ROC measurements at different locations: $\mu=698.27 \mu \mathrm{m}$ and $\sigma=0.33 \mu \mathrm{m}$.

The distribution is centered at $698.27 \mu \mathrm{m}$ and has a standard deviation of $0.33 \mu \mathrm{m}$. From Eq. 3, the factor $\gamma$ is given by

$$
\gamma=\frac{R^{\prime}}{R}=\frac{698.27}{700}=0.9975
$$

The uncertainty of this coefficient is obtained by simulation. The standard deviation of this distribution as well as the certified value sphericity error, $100 \mathrm{~nm}$, are used to set the coefficient in Eq. 8. The maximal amplitude $a_{0}$ is set to 0.025 and $N$ to 22. By simulating 100 times the 50 local ROCs practical measurements, the distribution of the average of the local ROCs is obtained, see Fig. 5. The uncertainty to take the average of the 50 local ROCs measurements as the value of the global ROC is taken to be the standard deviation, i.e. $0.05 \mu \mathrm{m}$. 


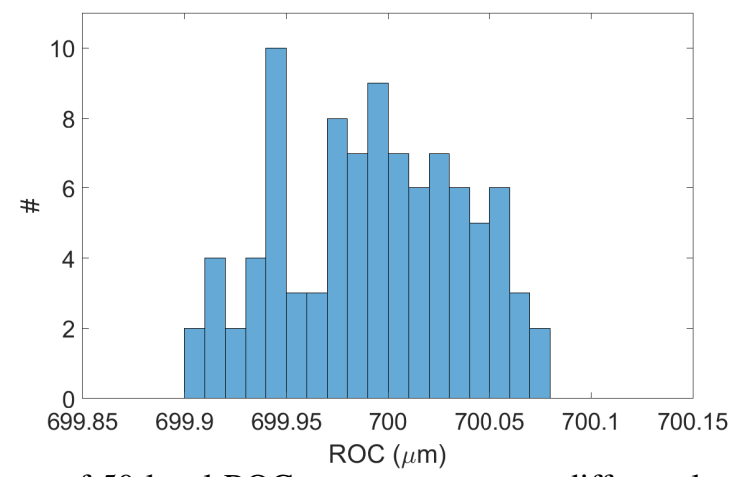

Figure 5: Distribution of the average of 50 local ROCs measurements at different locations: $\mu=700.002 \mu \mathrm{m}$ and $\sigma=$ $0.05 \mu \mathrm{m}$.

The uncertainty of the $\gamma$ coefficient is finally given by the addition of the uncertainty of the certified global ROC $R_{g}$ with the uncertainty of the average value of local ROC distribution $R_{m}$. Precisely,

$$
\frac{u(\gamma)}{\gamma}=\sqrt{\left(\frac{u\left(R_{g}\right)}{R_{g}}\right)^{2}+\left(\frac{u\left(R_{m}\right)}{R_{m}}\right)^{2}}=0.01 \%,
$$

which is 27 times better than with the traditional standards, see Eq. 5. From Eq. 3 this is also the uncertainty on ROC. It is worth noting that this uncertainty, mainly statistical, can be reduced if the number of measurements is increased. The uncertainty of $\kappa$ is also reduced to 0.0015 as well as the uncertainty which arises from fitting a spherical surface on an aspherical surface, see Fig. 6 . The maximal uncertainty of best sphere fit ROC becomes $0.022 \%$ (vs $0.28 \%$ ) which is still an improvement bigger than one order of magnitude.

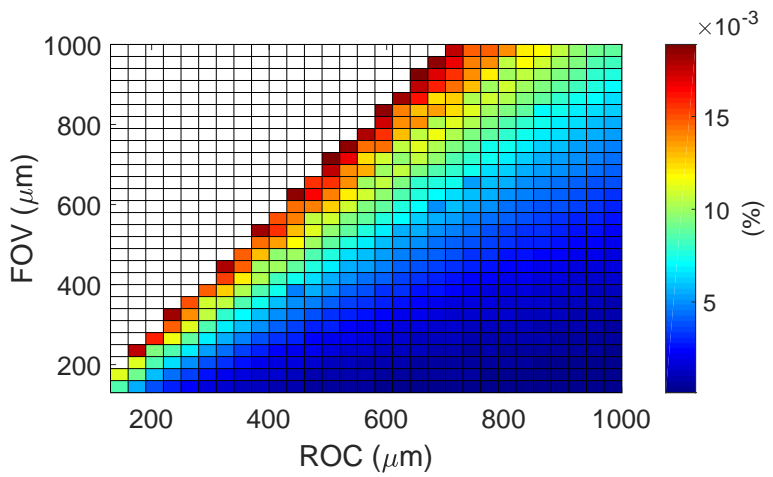

Figure 6: Uncertainty on ROC when performing a best sphere fit on an aspherical surface, $u(\kappa)=0.0015$.

\section{APPLICATION TO STANDARD CALIBRATION}

By obtaining the factor $\gamma$ with the local ROC measurements, there are now three ways to obtain the two coefficients $\alpha$ and $\beta$ necessary for the calibration: the step and periodic structure standards and the reference ball. In summary, the uncertainties of the different coefficients are

$$
\frac{u(\alpha)}{\alpha}=0.25 \%, \quad \frac{u(\beta)}{\beta}=0.075 \%, \text { and } \quad \frac{u(\gamma)}{\gamma}=0.01 \% .
$$


Thus this suggests using the ball measurements instead of the step standard to obtain $\alpha$. In this case, the uncertainty becomes, using Eq. 3,

$$
\frac{u(\alpha)}{\alpha}=\sqrt{\left(\frac{u(\gamma)}{\gamma}\right)^{2}+2\left(\frac{u(\beta)}{\beta}\right)^{2}}=0.11 \%,
$$

which increases the accuracy by a factor of 2.27. This demonstrates that this method is also well suited to calibrate an optical scanning profilometer regardless of the application.

\section{CONCLUSION}

A new calibration method for optical scanning profilometers is presented. It uses a reference ball whose global ROC is known with a high accuracy of $0.007 \%$. Measuring multiple local ROCs at random locations allows to benefit of this small uncertainty. With this approach, ROCs of aspherical and spherical surfaces are determined with a gain in accuracy over one order of magnitude. It also increases by a factor of more than 2 the vertical calibration confidence, decreasing the uncertainty from $0.25 \%$ to $0.11 \%$. This calibration method is thus believed to improve the characterization of spherical and aspherical microsurfaces and therefore, the quality of microelements such as microlenses.

\section{ACKNOWLEDGMENTS}

The authors want to thank A. Vetter and R. Kirner for fruitful discussions. This project has received funding from the European Union's Horizon 2020 research and innovation programme under grant agreement No 675745.

\section{References}

[1] P. J. de Groot, "Progress in the specification of optical instruments for the measurement of surface form and texture," 9110, p. 91100M, 2014.

[2] U. Griesmann, Q. Wang, J. Soons, and R. Carakos, "A Simple Ball Averager for Reference Sphere Calibrations," Proc. SPIE 5869 5869(3), p. 58690S, 2005.

[3] H. Gross, Handbook of Optical Systems Volume 1: Fundamentals of Technical Optics, vol. 1, Weinheim, wiley vch ed., 2005.

[4] B. N. Taylor and C. E. Kuyatt, "Guidelines for Evaluating and Expressing the Uncertainty of NIST Measurement Results,” NIST Technical Note 1297, p. 20, 1994.

[5] B. Hofmann-Wellenhof and H. Moritz, Physical geodesy, 2005. 